\title{
Inclinação Profissional e Personalidade: Estudo de Correlação entre Medidas desses Construtos
}

Professional Tendency and Personality: Research on the Correlation among Measures of these Constructs

Inclinación Profesional y Personalidad: Estudio de Correlación entre Medidas de estos Constructos
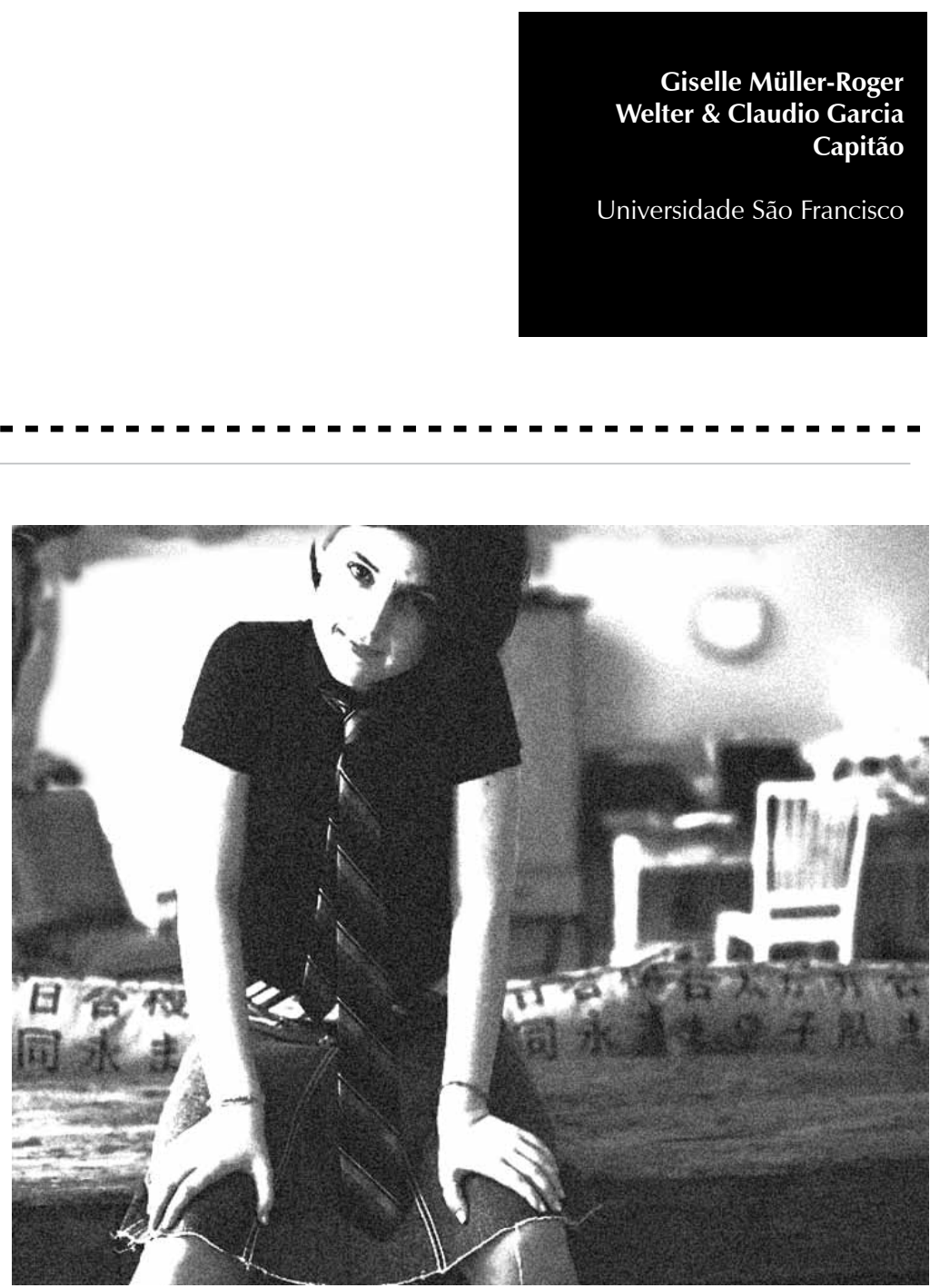
Resumo: Este trabalho pretende estudar a validade concorrente entre o 16PF - Questionário Fatorial da Personalidade e o BBT - Teste de Fotos de Profissões. O BBT é um teste projetivo, que se destina à apreensão do perfil de inclinação profissional, considerando oito fatores pulsionais. Apesar de o BBT ter sido concebido para adolescentes, estudos recentes mostraram que também pode ser empregado em adultos no contexto da orientação de carreira, coaching e seleção de pessoal. Enquanto o 16PF teve sua origem na análise fatorial, a construção do BBT se baseou nos pressupostos teóricos de Szondi sobre a estrutura pulsional das doenças mentais, com base em estudos clínicos com pacientes psiquiátricos. Embora os dois instrumentos partam de pressupostos teóricos muito diferentes, a estrutura fatorial do 16PF se assemelha em grande parte aos fatores pulsionais avaliados pelo BBT. Participaram do estudo 87 profissionais ( 35 homens e 52 mulheres) oriundos de diversas empresas, com idade média de 29,4 anos $(D P=8,2)$. Os resultados confirmaram as hipóteses de pesquisa no nível de significância 0,05.

Palavras-chave: Avaliação psicológica. Teste projetivo. Orientação profissional. Recursos humanos.

Abstract: This work has the presentation of the result of the convergent correlation research between 16PF - The Sixteen Personality Factor Questionnaire and the BBT - Profession Photos Test as objective. The BBT is a projective test destined to apprehend the vocational profile for the purpose of vocational guidance, considering eight drive needs or factors. Although the BBT was designed to be used with adolescents, recent work has shown that it is also useful in the context of career counseling coaching and selection of employees. While the 16PF had its origin in the factorial analysis, BBT's development is based on Szondi's theoretical framework, the drive theory of mental diseases, created upon clinical research with psychiatric patients. Although both instruments are based on very different theories, the factorial structure of the 16PF resembles largely to the drive factors evaluated in BBT. The participants were 87 employees ( 35 men and 52 women) derived from navigation companies and costal traffic, chemical and information technology segments, aged around 29,4 years $(S D=8,2)$. The results obtained in the correlation study confirmed the hypotheses to the convergence between both instruments at the significance level of 0,05.

Keywords: Psychological assessment. Projective test. Vocational guidance. Human resources.

Resumen: Este trabajo pretende estudiar la validez concurrente entre el 16PF - Cuestionario Factorial de la Personalidad y el BBT - Prueba de Fotos de Profesiones. El BBT es una prueba proyectiva, que se destina a la aprehensión del perfil de inclinación profesional, considerando ocho factores impulsivos. A pesar del BBT haber sido concebido para adolescentes, estudios recientes mostraron que también puede ser empleado en adultos en el contexto de la orientación de carrera, coaching y selección de personal. Mientras el 16PF tuvo su origen en el análisis factorial, la construcción del BBT se basó en los presupuestos teóricos de Szondi sobre la estructura de impulso de las enfermedades mentales, con base en estudios clínicos con pacientes psiquiátricos. Aunque los dos instrumentos partan de presupuestos teóricos muy diferentes, la estructura factorial del 16PF se asemeja en gran parte a los factores de impulso evaluados por el BBT. Participaron del estudio 87 profesionales ( 35 hombres y 52 mujeres) oriundos de diversas empresas, con edad Media de 29,4 años ( $\mathrm{DP}=8,2)$. Los resultados confirmaron las hipótesis de pesquisa en el nivel de significación 0,05

Palabras clave: Evaluación psicológica. Prueba proyectiva. Orientación profesional. Recursos humanos.

Cada vez mais, tem sido atribuída às características da personalidade e à motivação grande importância enquanto determinantes da competência e da produtividade dos indivíduos, e estão associadas tanto ao desempenho profissional como à realização pessoal (Cardozo, 2005; Chiavenato, 2004; Collins, 2001; Csikszentmihalyi, 1992; Drucker, 1996; Franco, 2001; Monicci, 2004; Sawickas, 2000; Seligman, 2004; Simonton \& Matthews-Simonton, 1987). A apreensão dos determinantes motivacionais e das características da personalidade também adquire grande importância para os indivíduos, na medida em que subsidia sua tomada de decisão em relação à escolha da profissão e ao desenvolvimento pessoal e profissional, considerando a tarefa, cultura e ambiente profissional. Ao mesmo tempo, embasa a contratação, a promoção e o treinamento de colaboradores por parte da empresa (Abrahão, 2000; Chiavenato, 2004; Dias, 2005; Franco; 2001). 
Essa dupla demanda está por trás da adoção de testes e avaliações da personalidade no âmbito da Orientação Profissional e da Psicologia Organizacional, fazendo com que os resultados influenciem cada vez mais, e de maneira decisiva, o destino de um número crescente de indivíduos (Abrahão, 2000; Bocato \& Bergel, 2005; Karpatschof \& Elkær, 2000). A qualidade dos testes empregados para esse fim adquire, portanto, grande relevância para as empresas, além influir na credibilidade dos instrumentos adotados nesse contexto. No Brasil, até o momento, há poucos instrumentos voltados para a apreensão das características da personalidade e da motivação no contexto organizacional, fazendo com que os profissionais que atuam nessa área recorram a instrumentos de avaliação da personalidade comumente usados no contexto clínico, adaptando-os às suas necessidades específicas. Uma outra prática bastante comum é a importação de instrumentos de avaliação da personalidade, que são traduzidos para o português (Bocato \& Bergel, 2005) e empregados sem serem antes submetidos à aprovação do Conselho Federal de Psicologia.

Uma das questões importantes a ser considerada em relação à avaliação psicológica no contexto organizacional diz respeito à desejabilidade social. Uma vez que o resultado obtido nos testes empregados tem impacto direto sobre o desenvolvimento profissional e da carreira do indivíduo, é comum que os testandos procurem parecer melhores do que são, respondendo da maneira que julgam mais condizente com as expectativas do empregador (Anastasi, 1975; Bartram, 1996; Dilchert, Ones, Visweswaran, \& Deller, 2006; Hammond \& Barrett, 1996; Meade, 2004; Van Kolck, 1973). Essa situação, que faz com que os usuários dos testes psicológicos no contexto organizacional recebam com desconfiança os resultados obtidos, contribuiu para o abandono do uso dos testes nesse âmbito, favorecendo, em contrapartida, a adoção de práticas mais intuitivas como entrevistas, assestment centers ou dinâmicas de grupo (Bocato \& Bergel, 2005; Cohen \& Swerdlik, 2005; Dias, 2005; Heggestad, 2006; Schuler, 2000).

O presente estudo se insere nesse panorama ao analisar dois testes amplamente utilizados no contexto da orientação profissional pela correlação entre o 16PF - Questionário dos 16 Fatores da Personalidade de Cattell (Cattell, 1989; Núñez \& Alemán, 2006, Russell \& Karol, 2002) e o BBT - Teste de Fotos de Profissões (Achtnich, 1991), trazendo em seu bojo não só questões de ordem teórica sobre a motivação e a personalidade, de natureza metodológica e de validade, mas uma reflexão acerca da correlação entre testes de natureza diferentes: um questionário (16PF) e um teste projetivo (BBT).

Entre as inúmeras definições de motivação encontradas na literatura (Allport, 1974; Cattell, 1975; Chiavenato, 2004; Herzberg, 1993; Maslow, 1987; Szondi, 1972), optou-se no presente estudo pela definição segundo a qual motivação é um "conjunto de fatores psicológicos (conscientes e inconscientes) de ordem fisiológica, intelectual e afetiva, que agem entre si e determinam a conduta do indivíduo" (Ferreira, 1986). A motivação expressa uma tensão persistente que leva o indivíduo a alguma forma de comportamento, visando à satisfação de uma ou mais necessidades. Necessidade é um estado de tensão ou desequilíbrio que resulta da falta, da ausência que sentimos dentro de nós mesmos sob a forma de mudanças fisiológicas. Essas mudanças, decorrentes do desequilíbrio no organismo, provocam atividades nervosas que permanecem atuantes no indivíduo até o restabelecimento do estado de equilíbrio, ativando-o no sentido da busca de satisfação 
dessa necessidade e levando-o a identificar, no meio ambiente, situações, ou meios, que favoreçam a sua satisfação. A afinidade com determinadas atividades permitem, assim, reduzir o estado de tensão decorrente do estado de desequilíbrio (Allport, 1974; Cattell, 1975; Maslow, 1987; Szondi, 1975).

Personalidade é um construto relativamente amplo, freqüentemente empregado para explicar tanto as diferenças individuais como a consistência do comportamento humano. Entre as maneiras de abordar a personalidade, destacam-se as teorias psicodinâmicas que atribuem papel central aos processos motivacionais ao buscar a compreensão do comportamento humano, entendendo que sua compreensão adequada só será possível a partir da personalidade total. A essa abordagem, contrapõe-se a teoria dos traços, segundo a qual estes seriam construídos gradativamente por meio da aprendizagem (Allport, 1974; Borg, 2001; Cattell, 1975, 1989; Deri, 1949; Szondi, 1965/1987, 1972).

Como expoente da teoria dos traços, Cattell (1975) considerava que há estruturas unitárias naturais na personalidade (traços), às quais atribuía valor causal enquanto fonte de variação na personalidade. Com base na análise fatorial, Cattell (Cattell, 1989; Núñez \& Alemán, 2006; Russell \& Karol, 2002) construiu o Teste 16PF, um questionário que tem por objetivo identificar os componentes primários da personalidade - ou seja, a maneira como pensa, percebe e se comporta, manifesta as suas atitudes, reações sociais e emocionais, bem como seus hábitos. Cada um dos 16 traços apreendidos nesse teste tem origem na relação entre as disposições internas e o processo de aprendizagem do indivíduo, e foram nomeados por meio de letras do alfabeto à medida que foram emergindo nos seus estudos (Tabela 1).
Tabela 1. Fatores primários da personalidade, segundo Cattell.

\begin{tabular}{ll}
\hline Fatores do 16PF \\
\hline A & Expansividade \\
B & Inteligência \\
C & Estabilidade emocional \\
E & Afirmação \\
F & Preocupação \\
G & Consciência \\
H & Desenvoltura \\
I & Brandura \\
L & Confiança \\
M & Imaginação \\
N & Requinte \\
O & Apreensão \\
Q1 & Abertura a novas experiências \\
Q2 & Auto-suficiência \\
Q3 & Disciplina \\
Q4 & Tensão \\
\hline
\end{tabular}

Fonte: Cattell, 1989; Núñez \& Alemán, 2006; Russell \& Karol, 2002

Como um dos teóricos da abordagem psicodinâmica, Szondi (1972, 1975, 1965/1987) considerava que as pulsões seriam fatores determinantes do destino humano, na medida em que influenciariam as escolhas do indivíduo em todos os níveis da sua existência, como a escolha do parceiro amoroso, das amizades, dos hobbies, do trabalho, da doença e da morte (quando esta é uma escolha). Estabeleceu o termo tropismo para designar o direcionamento da conduta, fazendo analogia com o reino vegetal, porém atribuindo-lhe novo significado, no sentido de determinação inconsciente das escolhas (Dorsch, 2001; Szondi, 1972). Ele postulava que a personalidade humana estaria estruturada sobre quatro vetores pulsionais, cada qual constituído de dois fatores polares (necessidades), que têm, por sua vez, orientação centrífuga e centrípeta (tendências), ou seja, uma orientação para fora e uma orientação para dentro: vetor sexual (princípio feminino e masculino), vetor 
paroxismal, (o processo psíquico por meio do qual o indivíduo é estimulado pelos afetos grosseiros e sutis), vetor do Ego (estrutura do Ego que faz a mediação entre as exigências subjetivas e as demandas externas), vetor do contato (agarrar-se, desprender-se do objeto, apegar-se e sair em busca do novo) (Benko, 1955; Borg, 2001; Deri, 1949; Szondi, 1972).

Com o objetivo de determinar o perfil pulsional, Szondi (1972) desenvolveu um teste projetivo que leva o seu nome, voltado para o psicodiagnóstico. Com base na teoria de Szondi, Achtnich (1991) desenvolveu o BBT - Teste de Fotos de Profissões, um teste projetivo para clarificação da estrutura pulsional da escolha profissional, voltado para a orientação profissional, considerando oito fatores de inclinação profissional (tabela 2).

Tabela 2. Fatores de inclinação segundo Achtnich (1991).

\begin{tabular}{l}
\hline Fator Orientação \\
\hline Fator W Suavidade \\
Fator $\mathrm{K}$ Força \\
Fator $\mathrm{S}$ Senso social \\
Fator Z Exposição \\
Fator $\mathrm{V}$ Razão \\
Fator $\mathrm{G}$ Espírito \\
Fator M Matéria \\
Fator O Oralidade
\end{tabular}

Comparando as teorias de personalidade de Cattell (1975) e de Szondi (1972), foram observadas semelhanças entre elas em muitos aspectos, tais como na determinação da conduta, na influência da hereditariedade, no número reduzido de fatores ou traços básicos, na polaridade dos fatores ou traços e na atribuição da fonte de energia por trás do comportamento ou impulso. No entanto, enquanto Szondi (1972, 1965/1987) chegou à descrição dos fatores a partir de suas observações clínicas, Cattell (1975) partiu da análise fatorial de termos que expressam traços da personalidade, sendo essa a diferença essencial entre ambos. As características dos fatores, ou traços, em ambos os modelos possuem semelhanças, havendo praticamente correspondência direta na descrição de muitos deles. A comparação entre os fatores concebidos por Szondi (1972), sua tradução no BBT (Achtnich, 1991) e o os fatores do 16PF (Cattell, 1989; Russell \& Karol, 2002), que se assemelham conceitualmente aos fatores szondianos, corresponde à hipótese de pesquisa do presente estudo.

Este estudo teve por objetivo verificar se há correlação entre os resultados obtidos em ambos os testes, considerando sua aplicação em adultos. Verificou-se a validade concorrente do BBT - Teste de Fotos de Profissões com o 16PF - Questionário dos 16 Fatores da Personalidade, uma vez que ambos parecem apreender traços semelhantes da personalidade.

\section{Método}

\section{Participantes}

Fizeram parte do estudo 87 profissionais de ambos os sexos, 35 homens e 52 mulheres, com idade média de 29,4 anos (DP 8,2), variando de 18 a 58 anos. Os participantes eram oriundos de três empresas diferentes do Estado de São Paulo, que compreendem os segmentos de navegação marítima, química e tecnologia da informação.

\section{Instrumentos}

BBT - Teste de Fotos de Profissões

O BBT- Teste de Fotos de Profissões (Achtnich, 1991) consiste de dois conjuntos de 96 fotografias, em branco e preto e em formato $10 \mathrm{~cm}$ por $10 \mathrm{~cm}$, retratando pessoas 
no desempenho de diferentes atividades profissionais, distribuídas em uma série masculina e feminina. Ao examinando, é solicitado que escolha as fotografias que lhe são simpáticas, antipáticas e indiferentes, de acordo com um critério estritamente pessoal. É dada a ele a orientação de que poderá escolher positivamente as fotos com as quais simpatizar, apesar de desconhecer a atividade ou não se identificar com a pessoa nela retratada. Embora o BBT tenha sido desenvolvido visando à aplicação individual, Achtnich (1979) também considerava a aplicação coletiva por meio da projeção das fotos no formato de diapositivos, para efeito de pesquisa. Os dados normativos do BBT no Brasil resultam dos estudos realizados por Jacquemin (2000) e Okino, Noce, Assoni e Pasian (2006) com estudantes secundaristas das redes pública e particular de ensino e com grupos pré-profissionais das áreas de exatas, humanas e biológicas, de ambos os sexos. Nos manuais são apresentados os valores medianos e os percentis referentes ao número de escolhas positivas, negativas e neutras no BBT-Br masculino (Jacquemin, 2000) e feminino (Okino et al., 2006), além das diferenças significativas na comparação dos fatores primários entre os grupos estudados.

\section{PF - Questionário Fatorial da}

Personalidade - 5a Edição

Segundo Russell e Karol (2002), o 16PF é um questionário destinado à faixa etária entre 15 e 65 anos, apresentado nas formas A, B, C, D e $E$, e, mais recentemente, na forma 16PF 5a edição, recomendado para aplicação em pessoas com ensino fundamental completo. O questionário é constituído de 16 escalas, contendo de 10 a 15 itens, o que perfaz um total de 185 itens. Cada item do questionário traz três alternativas de respostas. A realização do teste é feita por meio de lápis e papel, com duração aproximada de 60 minutos. A confiabilidade do 16PF foi obtida por meio do coeficiente Alfa de Cronbach, com valores que variaram entre 0,64 (Fator Q1) a 0,85 (Fator H), com uma média de 0,74. O coeficiente de confiabilidade obtido por meio de reteste considerou dois intervalos: duas semanas, com coeficientes que variaram entre 0,69 (fator $B$ ) e 0,86 (fator Q2) nos fatores primários, e de 0,84 e 0,91 nos fatores globais; dois meses, com coeficientes que variaram entre 0,56 (fator $L$ ) e 0,79 (fator $H$ ) nos fatores primários, e entre 0,70 e 0,82 nos fatores globais.

\section{Procedimento}

Após aprovação do projeto pelo Comitê de Ética, foram contatadas empresas do setor produtivo e de serviços para realização da coleta de dados. Após a apresentação dos objetivos e dos procedimentos da pesquisa aos representantes das empresas e da assinatura do termo de consentimento livre e esclarecido por todos os participantes, foi realizada a coleta de dados nas dependências das empresas. Inicialmente, foi aplicado o BBT, seguido do 16PF. A aplicação do BBT foi coletiva, por meio de diapositivos, com duração de 20 minutos. O tempo de exposição das fotos foi determinado eletronicamente e fixado em 6 segundos por foto. A aplicação do 16PF também foi coletiva, com duração média de 40 minutos, variando de 30 a 60 minutos, individualmente. No presente estudo, no BBT, foram consideradas apenas as somas absolutas das escolhas primárias positivas por fator, sendo que no 16PF foram considerados os escores brutos obtidos em cada fator.

\section{Resultados e discussão}

Para investigar as concomitâncias entre os fatores primários constituintes do BBT e os fatores primários do 16PF, foi realizada análise de correlação bivariada de Spearman (rho) bi-caudal (Tabela 3). Foram considerados significativos os valores que obtiveram nível de significância estatística menor do que 0,05, 
sendo muito significativos os que obtiveram níveis menores que 0,01 . Os valores acima de 0,05 foram considerados estatisticamente não significativos.

Tabela 3. Correlações (r) e níveis de significância (p) entre o BBT - Teste de Fotos de Profissões e o 16PF- Questionário Fatorial da Personalidade $(\mathrm{N}=87)$.

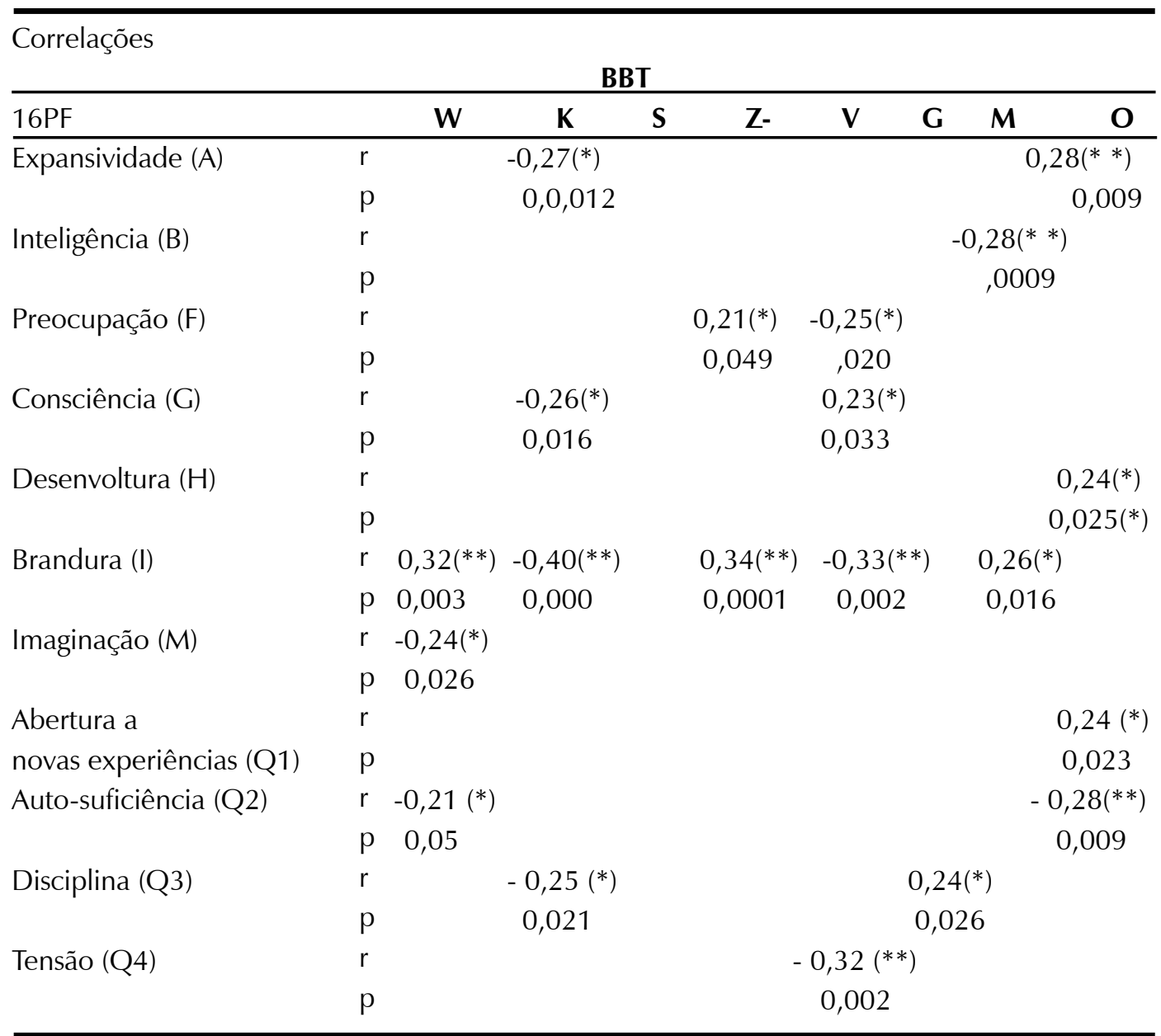

** Correlação muito significativa ao nível 0,01. * Correlação significativa ao nível 0,05.

A Tabela 3 sintetiza as concomitâncias entre os fatores pulsionais do BBT e os fatores da personalidade do 16PF, apresentando apenas as concomitâncias cujos valores do nível de significância estatística foram menores do que 0,05. A magnitude das correlações encontradas foi baixa, variando entre 0,24 e 0,33, provavelmente devido à diferença de estímulo dos dois testes.

Foram observadas sete correlações entre os fatores do BBT que correspondem ao vetor sexual szondiano (Szondi, 1972) e seis fatores do 16PF. O fator polar W (suavidade), que diz respeito à capacidade de empatia, receptividade e disponibilidade para atender e se adaptar às pessoas, apresentou três correlações significantes com o 16PF. A correlação positiva com o fator I brandura $(0,32)$ coincide com o construto de ambos, na medida em que o fator $I+$ do $16 \mathrm{PF}$ expressa o temperamento terno, sensível, dependente e indulgente, e o fator $W$ corresponde à necessidade de ternura e de proximidade psicológica, com disponibilidade para se adaptar ao outro. Já a correlação negativa com fator $M$ - intuição/sensação do 16PF $(-0,24)$, mostra que a 
polaridade negativa do fator $W$ corresponde a um sentido prático, guiado pelas sensações, expresso pelo fator $M$ - intuição/sensação (Achtnich, 1991; Cattell, 1989). Segundo Achtnich, o fator $W$ corresponde ao tato e à necessidade de tocar os objetos, de se adaptar ao outro e buscar a proximidade com ele, sugerindo correspondência entre os dois construtos. Observou-se, ainda, correlação negativa com o fator Q1 autosuficiência $(-0,21)$ do $16 \mathrm{PF}$, sugerindo que a polaridade negativa corresponde a uma tendência mais egocêntrica, em oposição à necessidade de manter um contato próximo, que corresponde ao construto de ambos os fatores (Achtnich, 1991; Cattell, 1989).

O fator $K$ (força) do BBT, polar ao fator $W$, diz respeito à necessidade de afirmação, expressa por meio do comportamento direto, dominante eagressivo em diferentes contextos. As correlações negativas encontradas nos fatores do 16PF, A - expansividade $(-0,27)$, G - consciência $(-0,26), I$ - brandura $(-0,40)$ e Q3 - disciplina $(-0,25)$, apontam esse construto. A correlação negativa do fator $K$ com o fator $A$ - expansividade expressa frieza, senso crítico, ceticismo, rigidez; a correlação negativa com o fator $G$ - consciência expressa falta de consideração pelos padrões morais do grupo e independência; a correlação negativa com o fator I (brandura) expressa falta de sentimentalismo, cinismo e dureza; a correlação negativa com o fator Q3 expressa tendência a deixar as pessoas à própria sorte, sentindo-se bem em ambientes desorganizados, com certa aversão por ambientes organizados e que requerem planejamento. Considerando que o fator $K$ expressa necessidade de ação e de transformação da realidade, a correlação negativa encontrada corresponde aos construtos de ambos os fatores (Achtnich, 1991; Cattell, 1989), na medida em que o comportamento disciplinado e organizado contrasta com a necessidade de agir com autonomia e com certa agressividade. As quatro correlações negativas encontradas entre ambos os testes expressam pouca capacidade de empatia, tendência a perseguir as próprias necessidades em detrimento das necessidades das outras pessoas e a rejeitar ambientes restritivos e disciplinadores, confirmando, assim, os construtos dos dois instrumentos.

Cabe destacar que o fator I - brandura do 16PF correlacionou-se com os fatores $W$ e $K$, sendo a correlação positiva com o fator $W$ e a negativa com o fator $K$, confirmando o construto subjacente ao vetor sexual, concebido por Szondi, segundo o qual ambos constituem polaridades.

No vetor paroxismal da teoria de Szondi (1972), que compreende os fatores polares $S$ (senso social) e $Z$ (expor) do BBT, não foram observadas quaisquer correlações relativas ao fator $S$, mas foram observadas duas correlações positivas do fator $Z$, com os fatores $F$ - preocupação $(0,21)$ e I brandura $(0,34)$. A correlação positiva com o fator $F$ expressa espontaneidade por meio de uma atitude despreocupada, alegre e animada, enquanto a correlação positiva com o fator I expressa afetação, tendência à dramatização, necessidade de chamar atenção, insegurança e necessidade de conquistar a simpatia das pessoas (Cattell, 1989). Os fatores do 16PF referem-se à dependência da aprovação externa, à necessidade de estar em evidência e de expor os próprios sentimentos, representados pelo fator $Z$ do BBT (Achtnich, 1991). Szondi atribuiu a esse fator características histéricas e exibicionistas.

No vetor do Ego da teoria szondiana (Szondi, 1972), foram observadas quatro correlações significantes entre o fator $V$ (razão) do BBT e os seguintes fatores do 16PF: $F-$ preocupação $(-0,25), G$ - consciência $(0,23)$, I - brandura $(-0,33)$ e Q4 - tensão $(-, 32)$. Essas correlações expressam o princípio da 
realidade e o predomínio da razão, com uma atitude mais sóbria, séria e retraída (F-), maior força do Superego $(\mathrm{G}+)$, tendência a seguir valores culturais convencionais (I-) e a adotar um comportamento mais relaxado e tranqüilo, paciente e com boa tolerância à frustração (Q4-). Essas correlações coincidem plenamente com o construto szondiano relativo ao fator $V$ (razão), que expressa o princípio da realidade e a necessidade de se adaptar à realidade ou de exercer controle sobre ela (Achtnich, 1991; Borg, 2001; Cattell, 1989; Núñez \& Alemán, 2006; Russell \& Karol, 2002; Szondi, 1972).

A correlação positiva observada em relação ao fator $G$ (espírito) do BBT foi com o fator Q3 - disciplina $(0,24)$, que expressa necessidade de planejar com antecedência (Russell \& Karol, 2002), o que pressupõe uma orientação para o futuro e o desconhecido por meio da elaboração de hipóteses, construto esse representado pelo fator $G$ do BBT (Achtnich, 1991). O fator disciplina se refere à necessidade de planejamento associada à necessidade de fazer as coisas corretamente e de maneira organizada. O fator $G$ aparece, portanto, associado a um dos aspectos presentes no fator Q3, a saber, a necessidade de prever e pensar o futuro, inerente ao planejamento.

Considerando as correlações do BBT relativas ao vetor do contato da teoria szondiana (Szondi, 1972) com o 16PF, observou-se que o fator $M$ (matéria) apresentou correlações com os fatores $B$ - inteligência $(-0,28)$ e $I$ brandura $(0,26)$. A correlação negativa entre o fator $M$ do BBT e o fator $B$ - inteligência do 16PF mostra-se especialmente interessante, na medida em que sugere que o pensamento concreto, característico do pragmatismo e da necessidade de ter contato direto com a matéria ou objeto de trabalho, representado pelo fator $M$ (matéria), estaria associado a menor capacidade cognitiva. Embora essa associação seja digna de uma análise mais aprofundada, o fato de o pensamento concreto aparecer associado ao fator $M$ sugere que a inteligência é medida como a capacidade de abstração e de manejar conceitos, estando, portanto, distante da realidade concreta, tangível e prática. A correlação positiva do fator $M$ do BBT com o fator brandura (I+) coincide com os construtos subjacentes aos dois testes, na medida em que I+ expressa, entre outros aspectos, a necessidade de apego e de segurança (Cattell, 1989), e o fator M (matéria) expressa a necessidade de segurança, por meio da retenção, da conservação e da estabilidade (Achtnich, 1991).

Já em relação ao fator $O$ (oralidade) do BBT, este se correlacionou positivamente com os fatores $A$ - expansividade $(0,28)$, $H$ - desenvoltura $(0,24)$ e com os fatores globais Q1 -abertura a novas experiências $(0,24)$, e negativamente com o fator Q2 - autosuficiência $(0,27)$ do 16PF. Essas correlações expressam abertura e alegria $(\mathrm{A}+)$, espontaneidade e expansividade $(\mathrm{H}+)$, uma atitude mais aberta e receptiva para o que vem ao encontro do indivíduo (Q1+), preferência por sentir-se cercado por pessoas e de fazer as coisas com os outros (Q2-), com tendência a mostrar-se dependente dos outros, corroborando a correlação entre os construtos de ambos os instrumentos (Achtnich, 1991; Cattell, 1989; Russell \& Karol, 2002).

Considerando todas as correlações encontradas entre o BBT e o 16PF, verificou-se que estas representam o mesmo construto latente. Cabe salientar, no entanto, que não foram observadas correlações relativas aos fatores $S$ (senso social) do BBT e aos seguintes fatores do 16PF: fator $C$ - estabilidade emocional, fator $E$ - afirmação, fator $L$ - confiança, fator $N$-requinte, fator $\mathrm{O}$ - apreensão. Como a maioria desses fatores expressa estados emocionais associados à auto-estima e à autoconfiança, era esperado que não fossem 
encontradas correlações com o BBT. A ausência de correlações do BBT com os fatores do 16PF que expressam ansiedade, auto-estima e autoconfiança coincide com a proposta de Achtnich (1991) ao desenvolver um teste que não tem por objetivo apreender aspectos relativos ao dinamismo psíquico. A tabela 4 apresenta as 20 correlações significativas encontradas entre o BBT e o 16PF, comparando-as aos vetores pulsionais szondianos e aos fatores de inclinação profissional descritos no BBT, que constituiu a hipótese de pesquisa do presente estudo.

Tabela 4. Comparações entre os fatores segundo Szondi, Achtnich e Cattell.

\begin{tabular}{|c|c|c|c|}
\hline \multicolumn{2}{|c|}{$\begin{array}{c}\text { Teste de Szondi } \\
\text { Szondi (1972) } \\
\text { Vetores e fatores pulsionais }\end{array}$} & $\begin{array}{l}\text { BBT } \\
\text { Achtnich (1991) } \\
\text { Fatores }\end{array}$ & $\begin{array}{l}\text { 16PF } \\
\text { Cattell (1989) } \\
\text { Fatores }\end{array}$ \\
\hline \multirow{2}{*}{ Sexual } & $\begin{array}{l}\mathrm{h}-\text { Eros } \\
\text { hermafrotidistmo } \\
(\mathrm{h}+)\end{array}$ & W - Suavidade & $\begin{array}{l}\text { Brandura }(\mathrm{I}+) \\
\text { Imaginação (M-) } \\
\text { Auto-suficiência (Q2-) }\end{array}$ \\
\hline & $\begin{array}{l}\mathrm{s} \text { - Thanatos } \\
\text { sadismo }(\mathrm{s}+)\end{array}$ & K - Força & $\begin{array}{l}\text { Expansividade (A-) } \\
\text { Consciência (G-) } \\
\text { Brandura (I-) } \\
\text { Disciplina (Q3-) }\end{array}$ \\
\hline \multirow[t]{2}{*}{ Paroxismal } & $\begin{array}{l}\text { e - Ética } \\
\text { epilepsia, sentido } \\
\text { ético }(\mathrm{e}+) \\
\text { E - Ética } \\
\text { epilepsia, acúmulo } \\
\text { de afetos (e-) }\end{array}$ & S- Senso social & --------------- \\
\hline & $\begin{array}{l}\text { hy - Moral } \\
\text { histeria (hy+) }\end{array}$ & Z-Exposição & $\begin{array}{l}\text { Preocupação }(\mathrm{F}+) \\
\text { Brandura }(\mathrm{I}+)\end{array}$ \\
\hline \multirow[t]{2}{*}{ Do Ego } & $\begin{array}{l}\text { k - Egosístole } \\
\text { catatonia (k-) }\end{array}$ & V - Razão & $\begin{array}{l}\text { Preocupação (F-) } \\
\text { Consciência (G+) } \\
\text { Brandura (I-) } \\
\text { Imaginação (M-) } \\
\text { Tensão (Q4-) }\end{array}$ \\
\hline & $\begin{array}{l}\text { P - Egodiástole } \\
\text { paranóia }(p+)\end{array}$ & G - Espírito & Disciplina (Q3+) \\
\hline \multirow[t]{2}{*}{ Do contato } & $\begin{array}{l}\text { D - Reter } \\
\text { depressão }(d-)\end{array}$ & M - Matéria & $\begin{array}{l}\text { Inteligência (B-) } \\
\text { Brandura (I+) } \\
\text { Auto-suficiência (Q2-) }\end{array}$ \\
\hline & $\begin{array}{l}M-\text { Apegar-se } \\
\text { mania }(m+)\end{array}$ & O - Oralidade & $\begin{array}{l}\text { Expansividade }(\mathrm{A}+) \\
\text { Desenvoltura }(\mathrm{H}+) \\
\text { Abertura a novas } \\
\text { experiências (Q1) }\end{array}$ \\
\hline
\end{tabular}

Muitos fatores do 16PF aparecem correlacionados com diferentes fatores do BBT: o fator $A$ (expansividade) se correlacinou negativamente com o fator $K$ (força) e positivamente com o fator $O$ (oralidade); o fator $F$ (preocupação) se correlacionou positivamente com o fator $Z$ (expor) e 
negativamente com o fator $V$ (razão); o fator $G$ - consciência do 16PF se correlacionaou negativamente com os fatores $K$ (força) e positivamente com o fator $V$ (razão) do $\mathrm{BBT}$, sendo que o fator $I$ - brandura se correlacionou com cinco fatores do BBT, com duas correlações negativas e três correlações positivas. O fator I - brandura do $16 \mathrm{PF}$, que corresponde às funções do eixo sentimento/pensamento, concebidos por Jung (Cattell, 1989), traz em seu bojo diferentes conceitos, que aparecem de maneira distinta na teoria de Szondi e no BBT (Achtnich, 1991; Szondi, 1972). A função sentimento, de Jung (brandura, I+) é caracterizada como a) empatia, sensibilidade, compaixão, ternura, necessidade de receber afeto, gentileza, indulgência, b) necessidade de segurança, apego e c) necessidade de atenção, hipocondria, afetação, tendência à dramatização. Os fatores correspondentes no BBT são, respectivamente, $W$ (suavidade), $M$ (matéria) e $Z$ (expor). O oposto da função sentimento, concebida por Jung (Cattell, 1989) é a função pensamento, que aparece associada aos fatores $K$ (força) e $V$ (razão) do BBT, sendo caracterizado como: a) falta de sentimentalismo, realismo, lógica, valores utilitaristas e b) dureza, cinismo, não indulgência, respectivamente. Essa observação dos fatores do 16PF sugere que o temperamento, a forma peculiar de participação nos diferentes contextos apreendida pelo 16PF, pode ser motivado por fatores de ordem mais profunda, fazendo com que o indivíduo "opte" inconscientemente por um determinado tipo de comportamento, tendo em vista a satisfação de suas necessidades (Allport, 1974; Cattell, 1975; Maslow, 1987; Seligman, 2004).

Não foram observadas correlações em relação aos seguintes fatores do 16PF: C - estabilidade emocional, E - afirmação, L - confiança, Nrequinte e $O$ - apreensão. A ausência dessas correlações sugere tratar-se de construtos diferentos daqueles apreendidos pelo BBT.
O único fator do BBT que não apresentou correlação com qualquer dos fatores do 16PF é o fator $S$ (senso social), que expressa compromisso ético, necessidade de ter uma participação social positiva por meio de ações de ajuda, assumindo responsabilidade frente à própria vida e aos outros e estabelecendo com eles uma relação caracterizada pela dependência dos outros frente à própria possibilidade para ajudá-los.

\section{Considerações finais}

A escassez de instrumentos para a avaliação psicológica voltados para o âmbito organizacional, a falta de credibilidade atribuída aos testes de personalidade, a crescente informatização dos instrumentos de avaliação psicológica e a definição de critérios para a elaboração, comercialização e uso dos testes psicológicos no Brasil pelo Conselho Federal de Psicologia (2003) estabelecem limites para o trabalho dos psicólogos, ao mesmo tempo em que estimulam o desenvolvimento, a atualização, a validação e a tradução de novos testes. Esse estímulo vai ao encontro da necessidade de se atender à crescente demanda por parte das empresas por instrumentos de avaliação psicológica que sejam confiáveis, em especial os voltados para a apreensão do perfil motivacional enquanto aspecto determinante da produtividade e da realização pessoal por meio do trabalho.

O presente estudo de correlação mostrou que há fortes indícios de que o BBT, apesar de ser um teste projetivo, apresenta muitas correlações com o 16PF, que é um questionário autodescritivo. Os resultados foram bastante interessantes, tendo sido encontradas 20 correlações entre ambos os testes, oito ao nível de significância 0,01 e doze correlações ao nível de significância 0,05, porém todas com baixa magnitude. Há, portanto, necessidade de novos estudos comparando esses instrumentos, visando a explorar as formas de apreensão da motivação, o que contribuiria para uma melhor descrição 
e compreensão do construto, a exemplo da correlação negativa encontrada entre o fator $B$ (inteligência) e o fator $M$ (matéria) do BBT.

Embora Cattell distinga claramente os conceitos traço e fator, sendo que o primeiro seria a expressão da combinação do segundo, observa-se que os fatores apreendidos pelo 16PF, a exemplo do fator $I$ - brandura, apresentam diferentes matizes fatoriais agrupados. As correlações encontradas com o BBT sugerem que este apreende matizes fatoriais básicos da personalidade, que podem ser combinados com maior ou menor intensidade, resultando nos fatores apreendidos no 16PF. Essa condição pode ser comparada com a Escala Pantone de Cores, na qual as diferentes cores e nuances são resultantes da combinação de algumas poucas cores primárias, que entram na sua composição em diferentes proporções. O mesmo parece ocorrer no âmbito da música, em que é possível compor uma infinidade de melodias por meio de novas combinações de apenas oito notas musicais.
A avaliação psicológica não é uma parte estática da ciência psicológica, mas envolve criatividade e espírito científico para explorar não só os instrumentos, mas também, e acima de tudo, o que eles representam, a apreensão das possibilidades humanas. A investigação do comportamento mediante instrumentos psicológicos faz uso de diferentes técnicas, apoiadas no tipo de avaliação que se pretende fazer. Cada instrumento, por sua vez, tem uma descrição metodológica específica, visando a garantir a precisão dos resultados obtidos, apoiados nas teorias que os suportam. Os grandes avanços das novas tecnologias e sua introdução crescente em diversas áreas da atividade humana também influenciaram a Psicologia, especialmente a área de avaliação psicológica. Esse fato exige que se invista no desenvolvimento de melhor qualidade dos instrumentos no contexto atual do trabalho e das organizações, além de buscar novas formas de aplicação, correção e emissão de parecer. 


\section{Giselle Müller-Roger Welter*}

Psicóloga, Especialista em Psicologia Escolar e Educacional, Mestre em Psicologia - Universidade São Francisco. Sócia-diretora GW Vocação \& Relações Humanas.

\section{Claudio Garcia Capitão}

Psicólogo, Especialista em Psicologia Clínica e Hospitalar, Pós-Doutorado em Psicologia Clínica PUC-SP. Docente dos Cursos de Graduação e de Pós-Graduação Stricto Sensu em Psicologia da Universidade São Francisco.

E-mail: cgcapitao@uol.com.br

\section{*Endereço para envio de correspondência:}

Rua São Benedito, 509, cj. 101 - São Paulo - SP - Brasil, CEP: 04735-000

E-mail: gwelter@gwconsult.com.br

Recebido 20/08/2008 Reformulado 28/01/2009 Aprovado 04/02/2009 


\section{Referências}

Abrahão, T. (2000, 28 de maio). Está faltando motivação? Folha de S. Paulo, São Paulo. Classificados Empregos.

Achtnich, M. (1979). Der Berufsbilder-Test - Projektives Verfahren zur Abklärung der Berufsneigung. Bern, Stuttgart, Wien: Verlag Hans Huber.

Achtnich, M. (1991). BBT - Teste de fotos de profissões. São Paulo: CETEPP.

Allport, G. W. (1974). Personalidade: padrões de desenvolvimento. São Paulo: EPU; Editora da Universidade de São Paulo.

Anastasi, A. (1975). Testes psicológicos: teoria e aplicação. São Paulo: EPU; EDUSP.

Bartram, D. (1996). The relationship between ipsatized and normative measures of personality. Journal of Occupational and Organizational Psychology, 69, 25-39.

Benko, A. (1955). Valores e limites do teste de Szondi. Revista de Psicologia Normal e Patológica, 1(2), 302-341. Separata.

Bocato, R., \& Bergel, M. (2005, 09 de outubro). Personalidade em teste. Folha de S. Paulo. Caderno Empregos.

Borg, J. G. (2001). Szondi's personality theory in the year 2000. Tampere: Pilot-Kustannus.

Cardozo, J. S. (2005, 10 de abril). Unir salário e satisfação. O Estado de São Paulo. Empregos.

Cattell, R. B. (1975). Análise científica da personalidade. São Paulo: IBRASA

Cattell, H. B. (1989). The 16PF: Personality in depth. Champaign: Institute for Personality and Ability Testing, Inc.

Chiavenato, I. (2004). Comportamento organizacional: a dinâmica do sucesso nas organizações. São Paulo: Thompson.

Cohen, R. J., \& Swerdlik, M. E. (2005). Psychological testing and assestment. New York: McGraw-Hill.

Collins, J. (2001). Empresas feitas para vencer - porque apenas algumas empresas brilham. Rio de Janeiro: Elsevier.

Conselho Federal de Psicologia. (2003a). Resolução $n^{\circ} 2 /$ 2003 - Define e regulamenta o uso, a elaboração e a comercialização de testes psicológicos e revoga a Resolução CFP $n^{\circ}$ 025/2001. Recuperado em 18 de setembro de 2005, de http://www.pol.org.br/legislacao/resolucoes. $\mathrm{cfm} ? \mathrm{ano}=2003$

Csikszentmihalyi, M. (1992). Flow - Das Geheimnis des Glücks. Stuttgart: Klett-Cotta.

Deri, S. (1949). Introduction to the Szondi test. New York: Grune \& Tratton.

Dias, A. (2005). Vista a camisa dos seus pontos fortes. Você $S$. A., $84,22-28$.

Dilchert, S., Ones, D. S., Visweswaran, C., \& Deller, J. (2006). Response distortion in personality measurement: born to deceive, yet capable of providing valid assessments? Psychology Science, 48(3), 209-225. Recuperado em 06 de outubro de 2006, de http://www.psychology-science. com/3-2006/ps_3_2006_209-225.pdf

Dorsch, F. (2001). Dicionário de psicologia. Petrópolis, RJ: Vozes.

Drucker, P. (1996). Administrando para o futuro. São Paulo: Pioneira.

Ferreira, A. B. H. (1986). Novo dicionário Aurélio da língua portuguesa (2a ed.). Rio de Janeiro: Nova Fronteira.

Franco, S. (2001). O profissionauta. São Paulo: Futura.

Hammond, S. \& Barrett, P. (1996). The psychometric and practical implications of the use of ipsative, forced-choice formal, questionnaires. The British Psychological Society: Occupational Psychology Conference. Book of Proceedings, $135-144$

Heggestad, E. D. (2006). Practical considerations for implementing personality testing in organizations. Symposium conducted at the Annual Meeting of the Society for Industrial and Organizational Psychology, Orlando, 12.

Herzberg, F. (1993). Motivation to work. New Brunswick: Transaction Pub.

Jacquemin, A. (2000). BBT-Br - O teste de fotos de profissões: normas - adaptação brasileira - estudos de caso. São Paulo: CETEPP.

Karpatschof, B. \& Elkær, H. K. (2000). Yet the bumblebee flies - The reliability of ipsative scores - Examined by empirical data and a simulation study. Research report. Department of Psychology, University of Copenhagen. Recuperado em 10 de outubro de 2005, de http://www.psy.ku.dk/forskning/ Udgivelser/Forskrap/forskrap1.pdf

Maslow, A. (1987). Motivation and personality. New York: Addison-Wesley.

Meade, A. W. (2004). Psychometric problems and issues involved with creating and using ipsative measures for selection. Journal of Occupational and Organizational Psychology, 77, 531-552.

Monicci, R. (2004). Motivação. Texto didático do curso de pósgraduação CEADE-PUCSP. São Paulo: PUCSP.

Núñez, M. A., \& Alemán, Y. (2006). El 16PF como instrumento psicométrico: una revisón de literatura. Recuperado em 25 de novembro de 2006, de http://ceci.uprm.edu/ aleman/16pf. html

Okino, E. T. K., Noce, M. A., Assoni, R. F., \& Pasian, S. R. (2006). $B B T-B r$ feminino: teste de fotos de profissões - adaptação brasileira, normas e estudos de caso. São Paulo: CETEPP.

Russell, M. T., \& Karol, D. L. (2002). 16PF: manual (5a ed. Atualizado e adaptado pelo Departamento de Estudos do CEPA). Rio de Janeiro: CEPA.

Sawickas, M. (2000). From career development to developing self through work and relationships. Prepared for presentation in Innovating Career Development Using Advances in Life Course and Lifespan Theory at the Annual meeting of the American Psychological Association, Washington, DC.

Schuler, H. (2000). Psychologische Personalauswahl - Einführung in die Berufseignungsdiagnostik. Göttingen: Verlag für Angewandte Psychologie.

Seligman, M. E. P. (2004). Felicidade autêntica: usando a nova psicologia positiva para a realização permanente. Rio de Janeiro: Objetiva.

Simonton, O. C., \& Matthews-Simonton, S. (1987). Com a vida de novo: uma abordagem de auto-ajuda para pacientes com câncer. São Paulo: Summus.

Szondi, L. (1972). Lehrbuch der experimentelle Triebdiagnostik. Band - Textband. Bern: Verlag Hans Huber.

Szondi, L. (1975). Introdução à psicologia do destino. São Paulo: Manole.

Szondi, L. (1965/1987). Schicksalsanalyse: Wahl in Liebe, Freundschaft, Beruf, Krankheit und Tod. Basel/Stuttgart: Schwabe \& Co AC.

Van Kolck, O. L. (1973). Técnicas de exame psicológico em suas aplicações no Brasil. Petrópolis, RJ: Vozes. 